\title{
Estratégias de Recrutamento de Participantes para Pesquisa Clínica: aspectos importantes e desafios
}

\author{
Mariana Salles La Terza Velleca ${ }^{1}$
}

\begin{abstract}
Resumo
Mesmo com um grande investimento de tempo e recursos por parte da equipe do Centro de Pesquisa e do patrocinador do estudo, estima-se que de $50 \%$ a $63 \%$ dos estudos não atingem a meta de recrutamento ou exigem uma extensão do prazo para atingí-la. Um baixo recrutamento de participantes pode levar a aumento de custos, diminuição do poder estatístico e atrasos na introdução de novos medicamentos, o que mostra a importância da adequada elaboração das estratégias de recrutamento, cujos aspectos práticos são apresentados nesta aula. As estratégias não devem ser planejadas apenas com o iminente início do estudo, mas sim durante todas as etapas que envolvem o acompanhamento do estudo no Centro de Pesquisa: etapa de avaliação da viabilidade, etapa de negociação contratual e orçamentária, etapa regulatória e, finalmente, antes do início do estudo com uma revisão do que já havia sido planejado e elaboração de Planos de Ação, tudo isso seguido de monitoramento contínuo durante todo o período de condução do estudo. Os principais desafios a serem enfrentados estão centrados na integração com os pacientes, com outros Centros de Pesquisa e integração entre a área técnica e de gestão. Apenas com dedicação e trabalho em equipe integrando todos os envolvidos é que se poderá atingir o sucesso do recrutamento e, consequentemente, contribuir para o sucesso do estudo.
\end{abstract}

Palavras chave: estratégias de recrutamento; pesquisa clínica; participantes de pesquisa

Velleca, Mariana Salles La Terza. "Estratégias de Recrutamento de Participantes para Pesquisa Clínica: aspectos importantes e desafios", in Anais do 20. Congresso Internacional Sabará de Especialidades Pediátricas

\section{Introdução}

Estima-se que aproximadamente um terço do tempo de condução dos estudos clínicos é dedicado ao recrutamento de participantes de pesquisa [8]. Uma pesquisa realizada em 2013 por uma consultoria americana de pesquisa clínica mostrou que, em média, laboratórios farmacêuticos alocam até $15 \%$ do orçamento total dos estudos em recursos para recrutamento de participantes, dependendo da fase de desenvolvimento do medicamento [5]. Mesmo com todo este investimento, a proporção de estudos que não atingem as metas de recrutamento ou que requerem um tempo estendido de recrutamento varia de $50 \%$ a $63 \%$ [2]. 
${ }^{1}$ Coordenadora de Pesquisa na Fundação José Luiz Egydio Setúbal. Farmacêutica graduada pela Faculdade Oswaldo Cruz, especialista em Pesquisa Clínica pela FCMSCSP e especializanda em Gestão de Projetos pelo Insper. e-mail: laterza.mariana@gmail.com

Um baixo recrutamento de participantes, como consequencia de falta de estratégias adequadas de recrutamento, pode resultar em tamanho amostral inadequado, o que reduz o poder estatístico do estudo; pode demandar em aumento de custos no caso de extensão do período de recrutamento ou implantação de novas estratégias; além disso, o estudo pode ser fechado sem ter dados suficientes que permitam uma adequada avaliação de eficácia e segurança dos medicamentos em teste, dificultando o lançamento de medicamentos inovadores que poderiam ser a solução para algumas doenças sem tratamento existente [4]. Tendo em vista a grande importância das estratégias de recrutamento de participantes de pesquisa, o objetivo principal desta aula é apresentar aspectos práticos para a elaboração destas estratégias, além de levantar tópicos para discussão e principais desafios enfrentados.

\section{Elaboração das estratégias de recrutamento}

Não existem intervenções padronizadas de recrutamento, pois cada estudo é único [2].

Normalmente a equipe do Centro de Pesquisa começa a pensar nas estratégias apenas com o iminente início do estudo, entretanto para um recrutamento eficiente este plano deve acompanhar todas as etapas de acompanhamento do estudo clínico no Centro de Pesquisa. Abaixo estão elencadas as principais etapas e os pontos importantes a serem levados em conta, referentes às estratégias de recrutamento.

- Etapa de avaliação de viabilidade da condução do estudo (feasibility): para não correr o risco de que a previsão de inclusão de pacientes seja mais otimista do que mostra, posteriormente, a realidade, o desenho do estudo deve passar por uma avaliação de viabilidade, questionando-se se os pacientes que preencherão os critérios de inclusão e exclusão do protocolo de fato existem na instituição [4].

- Etapa de negociação contratual e orçamentária: nesta etapa a equipe deve refletir sobre quais são as estratégias que poderiam auxiliá-los a alcançar a população-alvo do estudo e elencar quais são os recursos necessários para implantar estas estratégias, estimando os custos envolvidos a fim de negociar com o patrocinador do estudo.

- Etapa regulatória: durante a etapa de elaboração e submissão dos documentos para análise ética deve-se preparar o Plano de Recrutamento, descrevendo como os participantes serão abordados e convidados a participar do estudo. Esta é uma exigência da Norma Operacional no 001 de 2013 do Conselho Nacional de Saúde, a qual dispõe sobre a forma de submissão, avaliação e acompanhamento das pesquisas envolvendo seres humanos no Brasil e, em seu 
item 3.4.1, exige que este Plano seja submetido ao Comitê de Ética em Pesquisa (CEP) e Comissão Nacional de Ética em Pesquisa (CONEP), quando aplicável [3] .

- Antes do início do estudo: deve-se fazer uma reunião com toda a equipe para rever as estratégias que tinham sido previamente planejadas, estabelecer as ações para implantação de cada estratégia, definindo um prazo e um responsável para cada uma. Ferramentas de qualidade tais como a 5W2H podem ser utilizadas para ajudar com os Planos de Ação. Muitas são as estratégias que podem ser pensadas, dependendo do tipo, fase, desenho e critérios do estudo. Como exemplos, na tabela abaixo são apresentadas as estratégias e ações utilizadas pelo Centro de Pesquisa do Hospital Infantil Sabará para um estudo pediátrico de amigdalite, com recrutamento em Pronto-Socorro.

Tabela 1. Estratégias utilizadas em um estudo clínico pediátrico de amigdalite com recrutamento em Pronto-Socorro.

\begin{tabular}{|c|c|c|}
\hline Estratégias: & Engajamento de profissionais & Busca Ativa de participantes \\
\hline \multirow{6}{*}{ Ações: } & $\begin{array}{l}\text { Conversa diária com } \\
\text { profissionais }\end{array}$ & Busca em prontuário médico \\
\hline & \multicolumn{2}{|c|}{ Busca no Pré-Atendimento } \\
\hline & \multicolumn{2}{|c|}{ Cartaz sobre o estudo } \\
\hline & $\begin{array}{l}\text { Organização de café e } \\
\text { treinamento para profissionais }\end{array}$ & Encaminhamento Médico \\
\hline & Envio de e-mails lembrete & $\begin{array}{l}\text { Encaminhamento de outros } \\
\text { serviços }\end{array}$ \\
\hline & $\begin{array}{l}\text { Publicação de lembrete na Área } \\
\text { de Trabalho dos computadores } \\
\text { dos médicos }\end{array}$ & \\
\hline
\end{tabular}

A primeira estratégia planejada neste caso foi a Busca Ativa de participantes, e esta foi implantada através das seguintes ações:

Revisão dos prontuários médicos de pacientes identificados como potencialmente elegíveis de acordo com os critérios de inclusão e exclusão definidos pelo protocolo do estudo;

Acompanhamento das consultas de Pré-Atendimento (triagem) realizadas pela enfermagem a fim de identificar pacientes potencialmente elegíveis e sinalizar aos médicos para que confirmassem a eligibilidade; Exposição de cartazes de divulgação sobre o estudo no Pronto-Socorro; 
Encaminhamento médico;

Parceria com outros serviços de saúde localizados nas proximidades da instituição, para encaminhamento de pacientes potencialmente elegíveis.

Ao longo do período de recrutamento percebeu-se que os profissionais médicos e de enfermagem que não faziam parte da equipe do estudo, os quais eram essenciais para a identificação e encaminhamento de pacientes, não estavam devidamente motivados a contribuir para o recrutamento, uma vez que eles não se "sentiam parte” do estudo. Sendo assim, a segunda estratégia elaborada neste caso focava no engajamento profissional, não só dos membros da equipe do estudo, mas de todos os profisionais direta ou indiretamente envolvidos no recrutamento. Ações realizadas dentro desta estratégia:

Conversa diária com os profissionais, por telefone ou presencialmente, para recordá-los sobre a condução do estudo e esclarecer eventuais dúvidas;

Percebeu-se que a Busca Ativa no Pré-Atendimento também contribuía para o engajamento de profissionais, uma vez que a equipe do Centro de Pesquisa acompanhava as consultas de triagem junto com a enfermagem e aproveitava o momento para repassar os critérios do protocolo;

Cartazes de divulgação voltados aos profissionais também foram expostos nas áreas de Conforto Médico e de Enfermagem;

Foram organizadas sessões de café-da-manhã com treinamentos sobre o protocolo, mantendo-se o sigilo sobre os dados confidenciais, uma vez que nem todos os profissionais faziam parte da equipe do estudo;

Semanalmente eram enviados e-mails de lembrete sobre o estudo aos profissionais;

Foi postado na Área de Trabalho dos computadores dos médicos do Pronto-Socorro um lembrete sobre o estudo.

Como o intuito da aula é apresentar os aspectos práticos na elaboração das estratégias, não cabe aqui expor os resultados obtidos após a implantação das ações apresentadas acima. Outros exemplos poderiam ser citados, como a distribuição de folhetos e divulgação nas mídias sociais e website da instituição, que são ações que podem ser utilizadas dependendo do desenho do estudo e da população-alvo. Vale ressaltar que no caso de materiais de recrutamento que envolvam comunicação com os pacientes e familiares (folhetos, cartazes, texto para divulgação online,etc), estes devem ser previamente aprovados pelo CEP $[4,7]$.

\section{Sobre incentivos financeiros}

Embora muitos apontem os incentivos financeiros para participação em pesquisa clínica como uma questão polêmica e controversa, principalmente quando se trata de estudos pediátricos nos quais o 
fato de receberem uma quantia em dinheiro poderia funcionar como uma isca induzindo os responsáveis legais a consentir pela participação do filho no estudo, artigos e revisões sistemáticas publicados apontam que estes incentivos são muito eficientes para facilitar o recrutamento e melhorar a retenção de participantes [1]. No Brasil os incentivos apenas são permitidos na forma de reembolso de despesas de transporte e alimentação incorridas nos dias de procedimentos do estudo, desde que aprovado pelo CEP [4]. Nos EUA, por outro lado, a compensação financeira direta ou distribuição de brindes pela participação no estudo são aceitos, tendo o Food and Drug Administration (FDA) deixado a cargo dos CEPs a responsabilidade de determinar as quantias adequadas e não coercivas [1, 8].

\section{Monitoramento das estratégias de recrutamento}

Tão importante quanto a elaboração das estratégias é o monitoramento destas, a fim de avaliar o quanto estão sendo eficientes, se é necessário rever os Planos de Ação, e se as metas de recrutamento estão sendo atingidas.

Reuniões com a equipe são encorajadas, tendo sua periodicidade variada, de acordo com a

necessidade . É sempre importante lembrar que, quanto mais precoces forem as intervenções, maior a chance de maximizar o recrutamento [2].

Uma ferramenta de qualidade muito utilizada é o Diagrama de Ishikawa, para determinação de causa e efeito, seguido da elaboração de Planos de Ação.

\section{Principais desafios}

Pela experiência vivenciada em Centros de Pesquisa, pode-se afirmar que os principais desafios a serem enfrentados como forma de melhorar as possibilidades de recrutamento estão centrados na integração: integração com os pacientes, com outros Centros de Pesquisa e integração entre a área técnica e a de gestão.

Em relação à integração com os pacientes e familiares, a falta de conhecimento sobre o tema ainda é um empecilho importante, pois não está claro para a população em geral que a realização de estudos clínicos adequadamente conduzidos é essencial para o desenvolvimento e introdução de novos medicamentos, mais eficazes e/ou seguros que os já disponíveis [4]. Além disso, o acesso a informações detalhadas sobre os estudos em andamento e os resultados dos já finalizados pode ser difícil e complicado [8].

Outro grande desafio é a criação de redes de pesquisa clínica que fomentem o trabalho integrado entre os Centos de Pesquisa, como exemplo dos EUA, onde existem algumas redes focadas em doenças ou condições específicas. Levando em conta que a população pediátrica é menor e mais 
saudável do que a adulta , a criação de redes integrando Centros Pediátricos espalhados pelo país poderia contribuir para o recrutamento de participantes, pois aumentaria as chances de alcançar a população-alvo do estudo [8].

A integração da área técnica com a área de gestão é outro desafio a ser enfrentado pois, na maioria das vezes os funcionários dos Centros de Pesquisa, que são especializados em suas áreas de formação e Boas Práticas Clínicas, não dominam conceitos de marketing, por exemplo, que poderiam contribuir para a elaboração de campanhas de divulgação sobre o estudo. Outro exemplo é a falta de conhecimentos financeiros e de gestão para a adequada negociação com o patrocinador do estudo de recursos para recrutamento, correndo o risco de os custos envolvidos serem subestimados. A interface com outros setores da instituição que possam contribuir para a gestão dos estudos clínicos, principalmente no que diz respeito ao recrutamento de participantes, deve ser incentivada $[5,6]$.

\title{
6. Considerações finais
}

Diante do exposto cabe finalizar ressaltando que o recrutamento de pacientes é, e deve ser, um esforço mutidisciplinar. Não é papel só dos monitores /patrocinadores ou só da equipe do Centro de Pesquisa a preocupação com a inclusão de participantes no estudo. Apenas com dedicação e trabalho em equipe integrando todos os envolvidos é que se poderá atingir o sucesso do recrutamento e, consequentemente, contribuir para o sucesso do estudo.

\section{Recruitment strategies for clinical trials: challenges and important aspects}

\begin{abstract}
:
Even with a large investment of time and resources by the Research Center team and the sponsor, it is estimated that $50 \%$ to $63 \%$ of the studies failed to meet the recruitment target or requires an extension of the deadline for recruitment. A low participant recruitment can lead to increased costs , decreased statistical power and delays in the introduction of new drugs, which shows the importance of proper preparation of recruitment strategies, whose practical aspects are presented in this lesson . Strategies should be planned not only with the imminent start of the study, but during all steps involving the study at the Research Center: feasibility step, contractual and budget negotiation step, regulatory step and, finally, before the study begin with a review of what had already been planned and preparation of Action Plans, all of this followed by continuous monitoring throughout the period of conducting the study. The main challenges to be faced are focused on integration with patients with other research centers and integration between technical and management areas. Only with dedication and teamwork integrating all involved parts is that we can achieve the success of recruitment and, consequently, contribute to the success of the study.
\end{abstract}

Keywords: Recruitment Strategies. Clinical Trials. Research Subjects

\section{Referências}

[1] Bavdekar SB. Pediatric Clinical Trials. Perspect Clin Res. 2013 Jan-Mar; 4(1):89-99. 
[2] Bhatnagar S, Hoberman A, Kearney DH, et al. Development and Impact of an Intervention to Boost Recruitment in a Multicenter Pediatric Randomized Clinical Trial. Clinical Pediatrics. 2013 Oct; 53:151.

[3] BRASIL. Norma Operacional nº 001/2013. Dispõe sobre a organização e funcionamento do Sistema CEP/CONEP, e sobre os procedimentos para submissão, avaliação e acompanhamento da pesquisa e de desenvolvimento envolvendo seres humanos no Brasil, nos termos do item 5, do Capítulo XIII, da Resolução CNS nº 466 de 12 de dezembro de 2012. CONEP: Resoluções. 2014. Disponível em:

http://conselho.saude.gov.br/web_comissoes/conep/aquivos/CNS\%20\%20Norma\%20Operacional\%20 001\%20-\%20conep\%20finalizada\%2030-09.pdf

[4] Dainesi S. Como melhorar o recrutamento de pacientes em estudos clínicos? Rev Assoc Med Bras 2004; 50(3): 229-51.

[5] Evangelista E. Patient Recruitment: The Most Recognized Silent Driver of Clinical Development Costs. International Pharmaceutical Industry. 2013 vol.5(1).

[6] Jordan S, Morgan G. Recruitment to paediatric trials: a review. Welsh Paed J. 2011; 35:36-40.

[7] Laranjeira L, Barbosa LM, Guimarães HP. Estratégias de recrutamento em pesquisa clínica. Rev Bras Hipertens. 2008 vol.15(3):170-172.

[8] Tishler CL, Reiss NS. Pediatric Drug-Trial Recruitment: Enticement Without Coercion. Pediatrics. 2011;127;949. 